\title{
Migration of oral squamous cell carcinoma cells are induced by HGF/c-Met signalling via lamellipodia and filopodia formation
}

\author{
HIROKI YASUI ${ }^{1-3}$, YUICHI OHNISHI ${ }^{1,3}$, MASAHIRO NAKAJIMA ${ }^{3}$ and MASAMI NOZAKI ${ }^{1}$ \\ ${ }^{1}$ Department of Cell Biology, Research Institute for Microbial Diseases, Osaka University, \\ Suita, Osaka 565-0871; ${ }^{2}$ Graduate School of Dentistry, Osaka Dental University, Hirakata, Osaka 573-1121; \\ ${ }^{3}$ Second Department of Oral and Maxillofacial Surgery, Osaka Dental University, Hirakata, Osaka 573-1121, Japan
}

Received November 15, 2016; Accepted April 3, 2017

DOI: $10.3892 /$ or.2017.5587

\begin{abstract}
The activation of receptor tyrosine kinases (RTKs) results in cellular effects including cell proliferation, survival, migration and invasion; RTKs also play an important role in tumourigenesis. It has been reported that EGFR signalling controls the migration of oral squamous cell carcinoma (OSCC) SAS and HSC3 cells but not of HSC4 cells, although the proliferation of HSC4 cells is regulated by EGF/EGFR. In the present study, we investigated the roles of EGFR and the c-Met signalling pathway in cell migration via filopodia and lamellipodia formation, which may be prerequisites for migration. To explore the role of c-Met in cell migration, we inhibited c-Met RTK activity using the c-Met inhibitor SU11274 and activated c-Met using hepatocyte growth factor (HGF) in three OSCC cell lines HSC4, SAS and Ca9-22 and investigated migration potency using a wound healing assay. We showed that inhibition of c-Met significantly suppressed, and activation of c-Met significantly promoted, the migration of OSCC cells. Additionally, the migration of SAS and Ca9-22 cells was inhibited by the EGFR inhibitors AG1478 and cetuximab and promoted by EGF treatment. Moreover, migration potency was correlated with lamellipodia formation. Furthermore, western blot analyses demonstrated that SU11274 decreased and HGF increased lamellipodin protein levels as well as phosphorylated c-Met levels. Collectively, we demonstrated that c-Met signalling induced lamellipodia
\end{abstract}

Correspondence to: Dr Masami Nozaki, Department of Cell Biology, Research Institute for Microbial Diseases, Osaka University, 3-1 Yamadaoka, Suita, Osaka 565-0871, Japan

E-mail: mnozaki@biken.osaka-u.ac.jp

Abbreviations: HGF, hepatocyte growth factor; SCC, squamous cell carcinoma; OSCC, oral SCC; HNSCC, head and neck SCC; EGF, epidermal growth factor; EGFR, EGF receptor; RTK, receptor tyrosine kinase; DMEM, Dulbecco's modified Eagle's medium; FBS, fetal bovine serum; PBS, phosphate-buffered saline

Key words: epidermal growth factor, c-Met, cetuximab, AG1478, epidermal growth factor, SU11274, hepatocyte growth factor, lamellipodin, cell migration formation by upregulating lamellipodin, thereby promoting the migration of OSCC cells.

\section{Introduction}

Squamous cell carcinoma (SCC) accounts for most oral cancers (1). Despite improvements in diagnosis and treatment, oral SCC (OSCC) is still associated with a poor prognosis and high mortality rate $(2,3)$. One property of OSCC is that it is propagated by progressive local invasion $(4,5)$ and lymphatic metastasis, correlating with the clinical stage (6). Thus, a better understanding of the mechanisms of invasion is important for improving the treatments for OSCC. Aspects of tumour invasion include cell migration, tumour-stroma interactions at the invasive front and the influence of external stimuli, including growth factors (7-11).

Receptor tyrosine kinases (RTKs) mediate many fundamental effects in cells, including regulating migration (12). An example of RTK-activating growth factor is the epidermal growth factor (EGF) family, members of which act on the EGF receptor (EGFR) (13). Overexpression of EGFR has been detected in $\sim 90 \%$ of head and neck squamous cell carcinomas and correlates with the clinical stage (14) and EGFR is a factor influencing poor prognosis (15). The EGFR inhibitors, including the monoclonal antibody cetuximab and smallmolecule inhibitors (e.g., gefitinib, erlotinib and afatinib), have been used in the clinic to block signalling downstream of EGFR via ligand binding and ATP-binding sites of EGFR, respectively $(16,17)$.

Previously, we showed that EGFR inhibitors suppressed cell migration in OSCC SAS cells but not HSC4 cells $(18,19)$. Another RTK involved in the regulation of motility is c-Met, activated by hepatocyte growth factor (HGF, also known as scatter factor) $(20,21)$. c-Met is expressed in various cell types including epithelial and vascular endothelial cells, and its ligand HGF is released from stromal and some tumour cells $(22,23)$.

In migrating cells, filopodia and lamellipodia formation, by remodelling of the actin cytoskeleton, is observed at the leading edge of the moving side (24). Activation of the Rho family members Rac1 and Cdc42 via the PI3K/Akt or MEK/ERK pathway promotes the formation of lamellipodia and filopodia in prostate and breast cancers (25-27). 
c-Met/PI3K/Akt signalling plays a role in HGF-mediated lamellipodia formation and motility in lung endothelial cells (28). Furthermore, lamellipodin, a protein necessary for the formation of lamellipodia, has been reported to be important in cell migration via interactions with the Ena/VASP or Scar/WAVE complex $(29,30)$. Although Wnt signalling promotes formation of pseudopodia by the stimulating $\mathrm{Cdc} 42$ and RhoA in OSCC (31), other protein levels and signalling pathways necessary for the formation of pseudopodia in OSCC have yet to be determined.

In the present study, we examined the importance of EGFR and the c-Met signalling pathway in cell migration via filopodia and lamellipodia formation using OSCC cell lines.

\section{Materials and methods}

Cell culture and reagents. Three OSCC cell lines, HSC4, SAS and Ca9-22, were purchased from the RIKEN BioResource Center (Ibaraki, Japan). Cells were cultured in Dulbecco's modified Eagle's medium (DMEM), supplemented with $10 \%(\mathrm{v} / \mathrm{v})$ fetal bovine serum (FBS) at $37^{\circ} \mathrm{C}$ in a humidified atmosphere of $5 \% \mathrm{CO}_{2}$. DMEM and FBS were purchased from Gibco (Life Technologies, Tokyo, Japan). The antibodies used included anti-c-Met (Cell Signaling Technology, Tokyo, Japan), anti-phospho-c-Met (Tyr1234/1235, Cell Signaling Technology), anti-lamellipodin (Cell Signaling Technology), anti-cortactin (Santa Cruz Biotechnology, Santa Cruz, CA, USA) and anti- $\alpha$-tubulin (Sigma-Aldrich, Tokyo, Japan). Acti-stain $^{\mathrm{TM}} 488$ was purchased from Cytoskeleton, Inc. (Denver, CO, USA). Cetuximab (Erbitux ${ }^{\circledR}$ ) was purchased from Merck Serono, Co., Ltd. (Tokyo, Japan). SU11274 was from Sigma-Aldrich and AG1478 from Calbiochem (Merk Millipore, Tokyo Japan).

Scratch wound healing assay. Cell migration was determined using a scratch wound healing assay, as described elsewhere $(18,19)$ with slight modifications. Briefly, semi-confluent cells in 12-well plates were treated with $10 \mu \mathrm{g} / \mathrm{ml}$ mitomycin $\mathrm{C}$ for $4 \mathrm{~h}$ to block proliferation. The cells were subsequently wounded using a sterile $200-\mu 1$ pipette tip to generate a cell-free gap, $\sim 0.3 \mathrm{~mm}$ in width. Cells were then washed with phosphatebuffered saline (PBS) and photographed to record the wound width at $0 \mathrm{~h}$. One group of cells was cultured in DMEM with $10 \%$ FBS as a control, while the other groups were treated with various concentrations of cetuximab, AG1478, SU11274, EGF or HGF. After incubation, photographs were taken to evaluate migration.

Determination of lamellipodia. Cultured cells were fixed in $3.5 \%(\mathrm{w} / \mathrm{v})$ paraformaldehyde, permeabilized in $0.2 \%(\mathrm{v} / \mathrm{v})$ Triton X-100 and blocked in $2 \%(\mathrm{w} / \mathrm{v})$ bovine serum albumin (BSA). The cells were incubated with anti-cortactin antibody at $4^{\circ} \mathrm{C}$ overnight, followed by Alexa Fluor 594-conjugated IgG (Thermo Fisher Scientific, Yokohama, Japan) as the secondary antibody and Acti-stain 488 phalloidin (Cytoskeleton) for actin-fiber staining. After incubation, SlowFade gold antifade reagent with 4',6-diamidino-2-phenylindole (Invitrogen/ Life Technologies) was added to the cells. The specimens were observed by fluorescence microscopy (Olympus IX73; Olympus, Tokyo, Japan). We determined lamellipodia forma- tion by evaluating fluorescent actin fibers and cortactin co-localization at the cell periphery.

Western blotting. Cells were washed with PBS and then lysed in RIPA buffer consisting of $150 \mathrm{mM} \mathrm{NaCl}, 10 \mathrm{mM}$ Tris- $\mathrm{HCl}$, $\mathrm{pH} 8.0,1 \%(\mathrm{v} / \mathrm{v})$ Nonidet P-40, 0.5\% (w/v) deoxycholic acid, $0.1 \%(\mathrm{w} / \mathrm{v})$ SDS and $5 \mathrm{mM}$ EDTA with $1 \mathrm{X}$ Halt $^{\mathrm{TM}}$ Protease Inhibitor Cocktail (Thermo Fisher Scientific) and 1X Halt ${ }^{\mathrm{TM}}$ Protein Phosphatase inhibitor (Thermo Fisher Scientific). The protein concentrations of the lysates were determined using a $\mathrm{BCA}^{\mathrm{TM}}$ protein assay kit (Thermo Fisher Scientific) and equal amounts of protein were subjected to SDS-polyacrylamide gel electrophoresis. Separated proteins were transferred electrophoretically to Clear Trans PVDF membranes (Wako, Tokyo, Japan). Non-specific binding was blocked by incubation with 5\% (w/v) BSA in TBS/Tween-20 (TBS-T) for $1 \mathrm{~h}$ at room temperature. Membranes were probed with antibodies in TBS-T overnight at $4^{\circ} \mathrm{C}$ and then incubated with HRP-conjugated secondary antibodies. Antibody-antigen complexes were detected using ECL Plus Western blotting detection reagent (GE Healthcare, Little Chalfont, England).

Statistical analyses. Statistical analyses were performed using the one-way analysis of variance (ANOVA). Statistical significance $\left({ }^{*} \mathrm{P}<0.05,{ }^{* *} \mathrm{P}<0.01\right.$ and $\left.{ }^{* * *} \mathrm{P}<0.001\right)$ was evaluated using unpaired Student's t-test to assess the differences between the treated and the control samples.

\section{Results}

The migration potency of HSC4 cells is induced by serum stimulation and HGF/c-Met signalling. Previously, we showed that the EGFR pathway promoted migration of the OSCC cell line SAS but not the OSCC cell line HSC4. The migration potency of HSC4 cells was induced by stimulation of an unknown factor in serum other than EGF (19).

To assess whether the migration of other OSCC cells is regulated by the EGFR pathway, we investigated the influence of EGF and EGFR inhibitors on the migration of the gingival cancer cell line Ca9-22. The migration activity of Ca9-22 cells, as well as SAS cells, was decreased significantly by cetuximab treatment at $20 \mu \mathrm{g} / \mathrm{ml}$ (Fig. 1A) and AG1478 treatment at $5 \mu \mathrm{M}$ (Fig. 1B) but was enhanced significantly by EGF addition at $100 \mathrm{ng} / \mathrm{ml}$ (Fig. 1C). Additionally, migration of HSC4 cells was unaffected by the addition of EGFR inhibitors or EGF (Fig. 1). However, the addition of serum induced the migration of HSC4 cells significantly, as it did with SAS and Ca9-22 cells (Fig. 2A). These results indicate that cell migration was induced by (a) serum component(s) other than EGF among the OSCC cell lines examined.

Next, we investigated the effects of c-Met on cell migration potency using a scratch wound healing assay in OSCC cell lines, because c-Met is an RTK involved in cell migration along with EGFR $(20,32)$. When cells were treated with the c-Met inhibitor SU11274 at $10 \mu \mathrm{M}$, the migratory potency of all cell lines was reduced significantly (Fig. 2B). Moreover, the migratory activity of all cell lines was enhanced significantly by addition of the c-Met ligand HGF at $20 \mathrm{ng} / \mathrm{ml}$ (Fig. 2C). These results suggest that the migration of HSC4 cells is regulated by $\mathrm{HGF} / \mathrm{c}-\mathrm{Met}$ signalling but not EGF/EGFR signalling. 

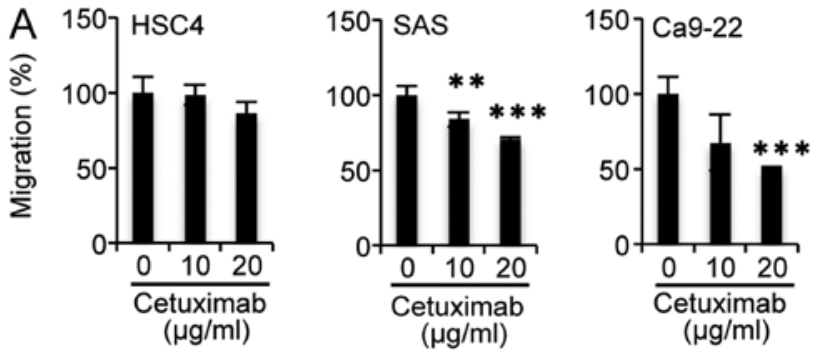

$(\mu \mathrm{g} / \mathrm{ml})$
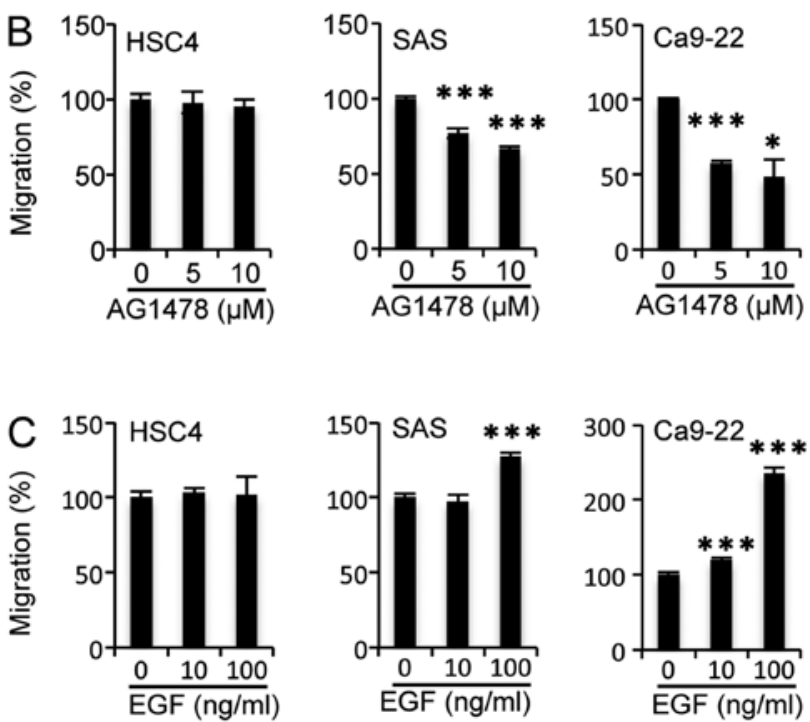

Figure 1. EGF/EGFR signalling induces the migration potency of SAS and Ca9-22 cells, but not HSC4 cells. Wound-healing assay in HSC4, SAS and Ca9-22 cells treated with (A) cetuximab, (B) AG1478 or (C) EGF. The widths of the cell scratches were measured after 0 and $12 \mathrm{~h}$ of culture using ImageJ software. The migration percentage was calculated as the mean width of the cell scratch. The effects of cetuximab, AG1478 and EGF treatment on cell migration were investigated by comparing the scratch widths between treated and untreated cells; the width in the untreated was set at $100 \%$. Bars represent the means \pm SEMs. One-way ANOVA was used. ${ }^{*} \mathrm{P}<0.05 ;{ }^{* *} \mathrm{P}<0.01$; ${ }^{* * * *} \mathrm{P}<0.001$.

Furthermore, both EGF/EGFR and HGF/c-Met signalling play a role in the migration of SAS and Ca9-22 cells.

Filopodia and lamellipodia formation is induced by EGFR signalling in OSCC SAS cells but not HSC4 cells. Cell migration is controlled by dynamic remodelling of the actin cytoskeleton. In this process, the formation of pseudopodia, including filopodia and lamellipodia, consisting of actin fibres at the leading edge of the cells plays a pivotal role (33). To understand the relationship between the formation of filopodia and lamellipodia and EGFR signalling in OSCC cells, we examined the effects of EGFR inhibitor AG1478 and EGF treatment on filopodia and lamellipodia formation in migrating cells facing the scratch wound. Filopodia appeared in the untreated control and AG1478-treated HSC4 and SAS cells by $0.5 \mathrm{~h}$. Cells with filopodia increased in both cell lines for over $1 \mathrm{~h}$. After $12 \mathrm{~h}$, some filopodia formation was observed in almost all control and AG1478-treated HSC4 cells (Fig. 3A). However, although numbers of the cells with filopodia increased after $12 \mathrm{~h}$ in SAS control cells, the proportion of cells with filopodia was $\sim 50 \%$ after $12 \mathrm{~h}$ of AG1478 treatment compared with the control (Fig. 3A).
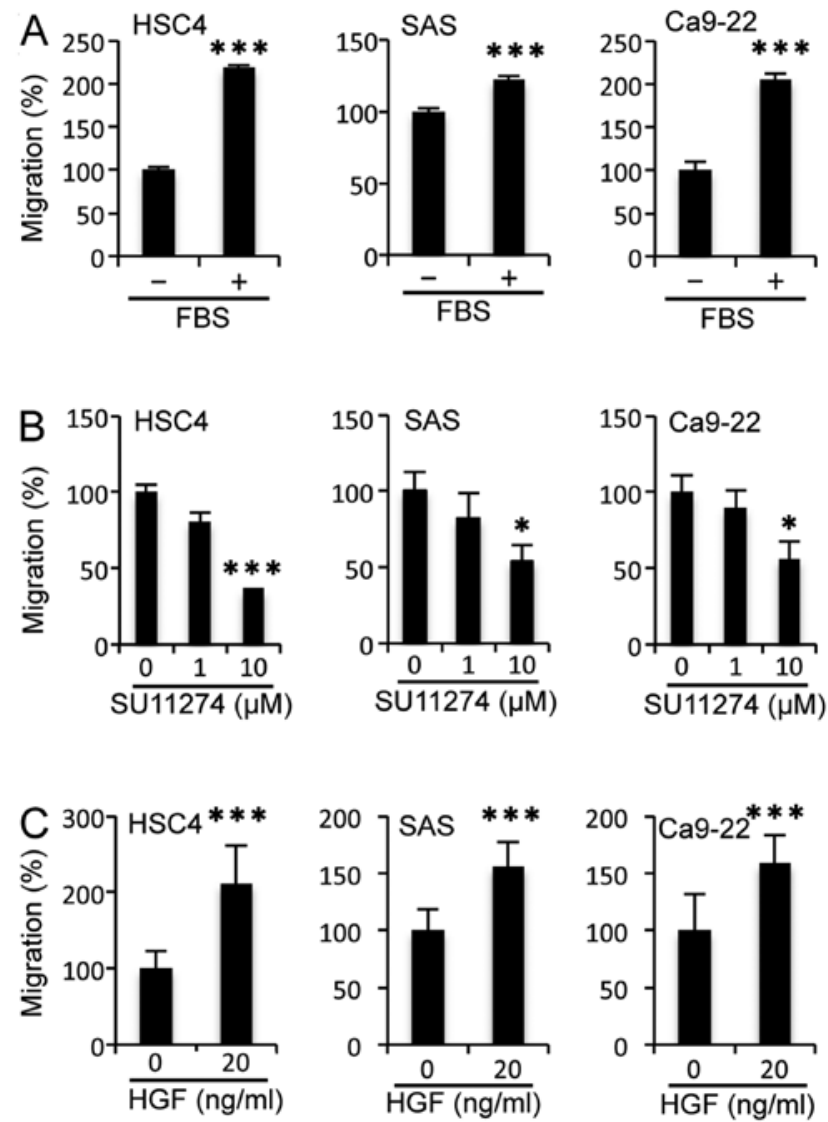

Figure 2. Migration potency of HSC4 cells is induced by FBS or HGF/c-Met signalling. Wound-healing assay in HSC4, SAS and Ca9-22 treated with (A) FBS, (B) SU11274 and (C) HGF. The widths of the cell scratches were measured after 0 and $12 \mathrm{~h}$ of culture using Image J software. The migration percentage was calculated as the mean width of the cell scratch. The effects of FBS, SU11274 and HGF treatment on cell migration were investigated by comparing the scratch width between treated and untreated cells; the width in the untreated was set at $100 \%$. (A and C) Bars represent the means \pm SDs Student's t-test was used. ${ }^{* * *} \mathrm{P}<0.001$. (B) Bars represent the means \pm SEMs. One-way ANOVA was used. ${ }^{*} \mathrm{P}<0.05 ;{ }^{* * *} \mathrm{P}<0.001$

We next examined the effects of EGF on filopodia formation. In HSC4 and SAS cells, filopodia had formed in $~ 15-20 \%$ by $0.5 \mathrm{~h}$ and in majority by $12 \mathrm{~h}$ regardless of EGF treatment (Fig. 3B). These results showed that EGFR signalling was necessary for filopodia formation in SAS cells but not in HSC4 cells and that addition of EGF had no apparent effect on filopodia formation.

Approximately $40 \%$ of untreated HSC 4 and $80 \%$ of untreated SAS cells formed lamellipodia by $12 \mathrm{~h}$. The ratio of lamellipodia formed in SAS cells treated with AG1478 was significantly lower than that in control SAS cells after $6 \mathrm{~h}$. However, lamellipodia formation was not affected in AG1478 treated HSC 4 cells at $12 \mathrm{~h}$ (Fig. 3C). EGF increased the number of SAS cells with lamellipodia, compared with the control and had not apparent effect on HSC4 cells (Fig. 3D). These results suggest that the EGFR pathway is involved in filopodia and lamellipodia formation in SAS cells but not in HSC4 cells.

Filopodia and lamellipodia formation is induced by HGF/c-Met signalling in HSC4 cells and SAS cells. We evaluated the effects of $\mathrm{HGF} / \mathrm{c}-$ Met on filopodia and lamellipodia 
A

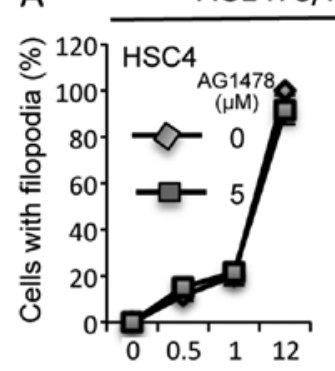

Culture periods $(\mathrm{h})$

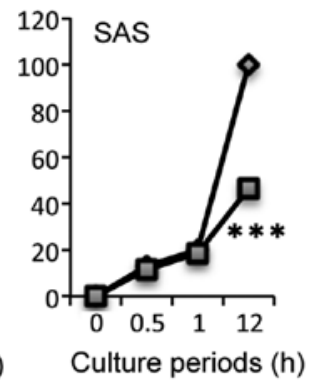

B

EGF/filopodia

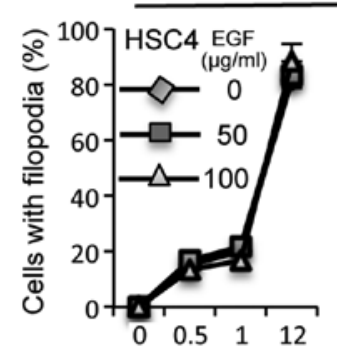

Culture periods $(\mathrm{h})$

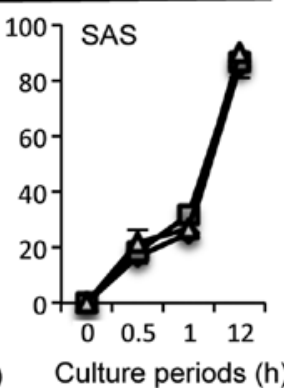

C

AG1478/lamellipodia
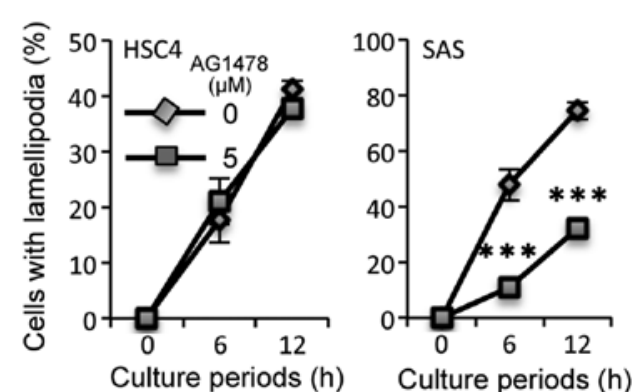

D

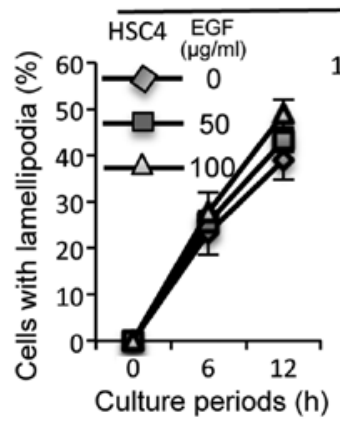

EGF/lamellipodia

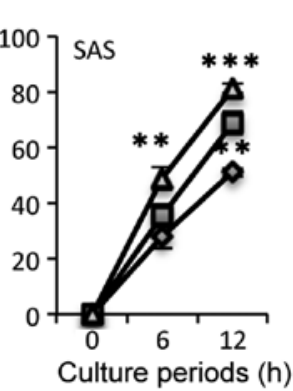

Figure 3. Lamellipodia formation is induced by EGF/EGFR signalling in OSCC SAS cells. (A) AG1478 inhibited filopodia formation in SAS cells but not in HSC4 cells. (B) EGF did not affect filopodia formation in HSC4 or SAS cells. (C) AG1478 inhibited lamellipodia formation in SAS cells but not in HSC4 cells. (D) EGF induced lamellipodia formation in SAS cells but not in HSC4 cells. Cells were grown in 96-well plates overnight: confluent cells were scratched and then treated with various reagents. The percentages of cells forming filopodia and lamellipodia were determined in the field of view using phase contrast microscopy. Graphs show means \pm SEMs. One-way ANOVA was used. ${ }^{* * *} \mathrm{P}<0.01 ;{ }^{* * *} \mathrm{P}<0.001$.
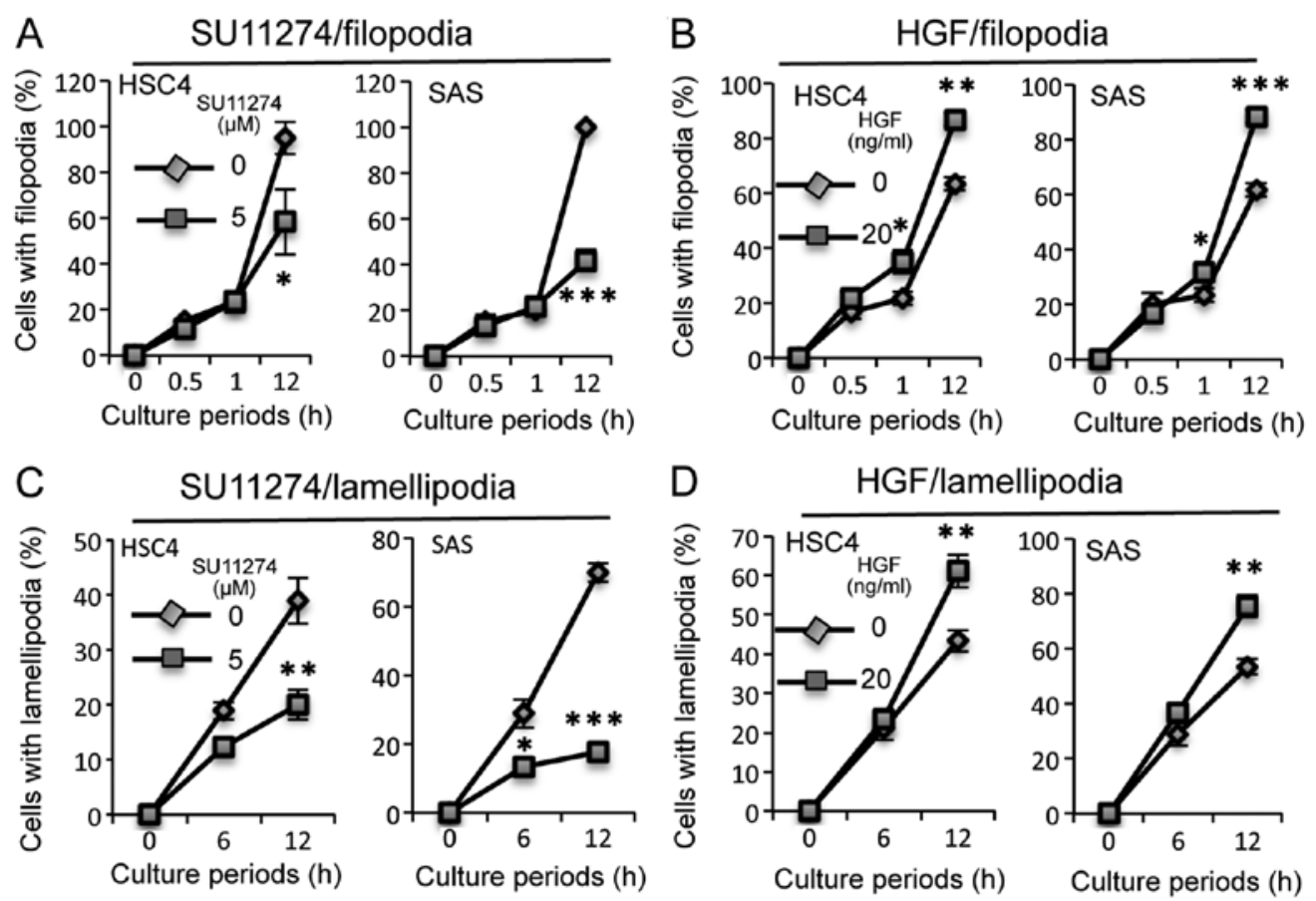

Figure 4. Filopodia and lamellipodia formation are induced by HGF/c-Met signalling in HSC4 and SAS cells. SU11274 inhibited filopodia (A) and lamellipodia (C) formation in HSC4 and SAS cells. HGF promoted (B) filopodia and (D) lamellipodia formation in HSC4 and SAS cells. Cells were grown in 96-well plates overnight; confluent cells were scratched and treated with various reagents. The percentages of cells forming filopodia and lamellipodia were determined in the field of view using phase contrast microscopy. Graphs show means \pm SEMs. One-way ANOVA was used. ${ }^{*} \mathrm{P}<0.05 ;{ }^{* * *} \mathrm{P}<0.01 ;{ }^{* * *} \mathrm{P}<0.001$.

formation in controlling migration potency in HSC4 and SAS cells. Filopodia formation in HSC4 and SAS cells treated with SU11274 did not differ from that in the untreated cells after 0.5 and $1 \mathrm{~h}$ of culture. However, filopodia formation was 


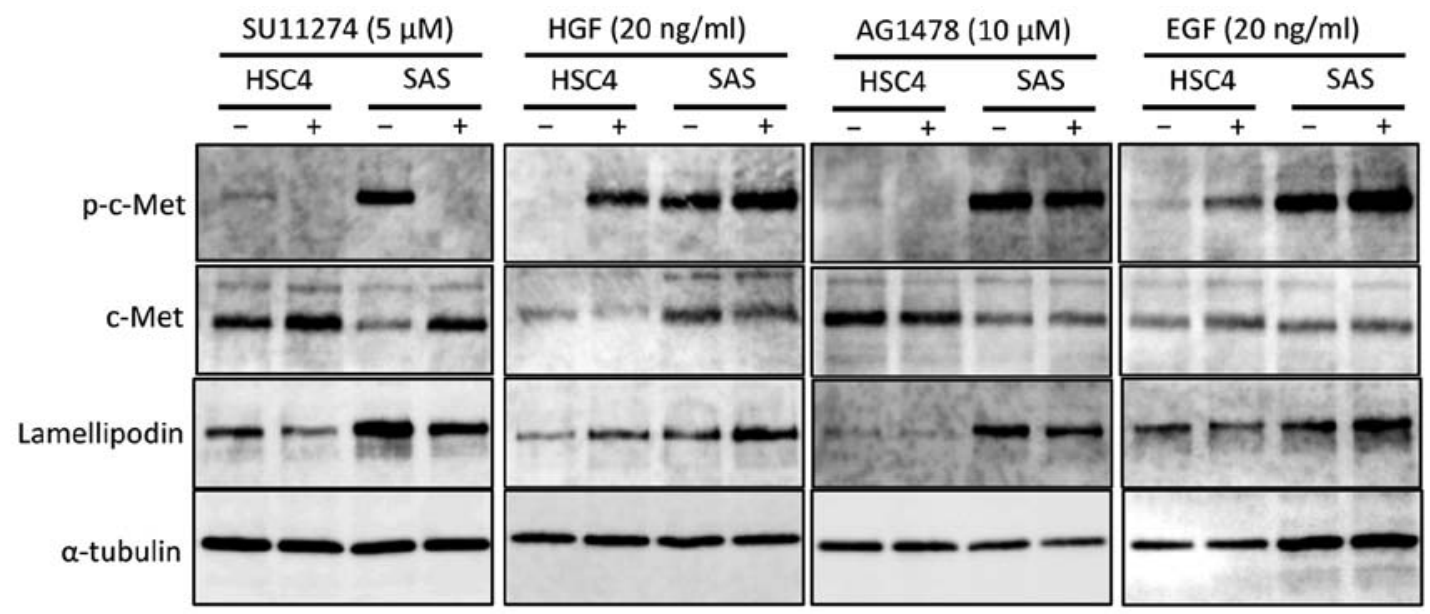

Figure 5. Lamellipodin is regulated by HGF/c-Met signalling but not by EGF/EGFR signalling, although c-Met phosphorylation is regulated by EGF/EGFR signalling in HSC4 cells. Cells were treated with SU11274, HGF, AG1478 and EGF. Western blotting was performed to determine the phosphorylation levels of c-Met (using anti-c-Met and anti-phospho-c-Met antibodies) and protein level of lamellipodin (using anti-lamelliodin antibody). $\alpha$-tubulin was used as a loading control.

reduced significantly compared with untreated cells after $12 \mathrm{~h}$ of SU11274 treatment (Fig. 4A).

Because filopodia formation in HSC4 and SAS cells was decreased by inhibition of c-Met signalling, we next examined the effects of HGF, the ligand of c-Met. Filopodia-forming HSC4 and SAS cells were increased after $1 \mathrm{~h}$ of HGF treatment compared with the serum-free medium (Fig. 4B). Additionally, the ratios of HSC4 and SAS cells with lamellipodia was reduced significantly by SU11274 treatment for 6-12 h compared with untreatment cells (Fig. 4C). Furthermore, the percentages of lamellipodia-forming HSC4 and SAS cells after HGF treatment did no differ from those of untreated cells after $6 \mathrm{~h}$ but were increased after $12 \mathrm{~h}$ (Fig. 4D). These results suggest that HGF/c-Met signalling is important for the formation of filopodia and lamellipodia in HSC4 and SAS cells, and that EGFR signalling plays an important role in filopodia and lamellipodia formation in SAS cells.

Lamellipodin is regulated by $\mathrm{HGF} / \mathrm{c}$-Met signalling but not by EGF/EGFR signalling, although c-Met phosphorylation is regulated by EGFR signalling in HSC4 cells. To further elucidate the role of HGF/c-Met and EGF/EGFR signalling involved in the formation of lamellipodia in the OSCC cell lines examined, we focused on the lamellipodin protein, which is related to the formation of lamellipodia at the leading edge, together with Ena/VASP proteins (34). We found that when the phosphorylation level of c-Met was decreased by SU11274 treatment, the lamellipodin level was also decreased in both HSC4 and SAS cells. Lamellipodin levels and c-Met phosphorylation were both upregulated by HGF stimulation in both HSC4 and SAS cells. Furthermore, inhibition of EGFR signalling by AG1478 decreased c-Met phosphorylation and lamellipodin levels in SAS cells. However, although the phosphorylation level of c-Met was reduced by AG1478, the level of lamellipodin was not changed in HSC4 cells. Additionally, the levels of c-Met phosphorylation and lamellipodin protein were increased by EGF stimulation in SAS cells. In contrast, although c-Met phosphorylation was increased by EGF stimulation, lamellipodin was unaffected in HSC4 cells.
These results suggest that EGFR signalling increases the level of lamellipodin protein via a process involving of c-Met, and that this promotes the formation of lamellipodia in SAS cells. EGFR signalling could lead to phosphorylation of the c-Met, but it does not affect the level of lamellipodin in HSC4 cells (Fig. 5).

\section{Discussion}

It has been reported that EGFR and c-Met are involved in cell migration (20,35-38). In the present study, we showed that migration of the OSCC cell lines SAS and Ca9-22 was regulated by both the EGFR and c-Met signalling pathways. In contrast, migration of OSCC HSC4 cells involves c-Met activation but not the EGFR pathway. Indeed, HSC4 cell migration was resistant to EGFR inhibitor and sensitive to a c-Met inhibitor. The mechanisms of EGFR inhibitor resistance have been classified into two categories: developing of a secondary mutation in EGFR and bypassing or activation of an alternative pathway (39). We demonstrated previously that the proliferation of HSC4 cells is sensitive to an EGFR inhibitor (18). Treatment with the EGFR inhibitor AG1478 decreased phosphorylation levels of EGFR, AKT and ERK, as reported previously. These findings indicated that the EGFR pathway plays a distinct role from that of the c-Met pathway in HSC4 cells. c-Met played an important role in the migration of all OSCC cell lines examined, suggesting that c-Met may be an appropriate therapeutic target for invasion and metastasis.

In the present study, we showed that the HGF/c-Met pathway plays an important role in the formation of lamellipodia and filopodia, as well as in the migration of OSCC cells. These results are consistent with previous reports that $\mathrm{HGF} / \mathrm{c}-$ Met signalling promoted cell migration through lamellipodia and filopodia formation in lung endothelial cells (28) and in some normal cells (30). Thus, it is possible that lamellipodia and filopodia formation is regulated by c-Met signalling, thereby promoting the migration of OSCC cells. ERK and PI3K/Akt serve as downstream effectors of c-Met signalling (28). However, the 
molecules downstream of c-Met/ERK and c-Met/PI3K/Akt signalling that directly regulate filopodia and lamellipodia formation remain unknown. In this context, we showed that c-Met signalling was involved in the regulation of lamellipodin protein levels. Promotion of cell migration potency via the c-Met pathway is possibly regulated by increasing the level of lamellipodin, because upregulation of lamellipodin protein markedly promoted cell migration (30).

In this study, filopodia formation in SAS was inhibited by an EGFR inhibitor, but was not promoted by EGF. These data suggest that filopodia formation is regulated by ligandindependent EGFR signalling or by EGFR ligands other than EGF. Ligand-independent activation of EGFR, including Src-mediated integrins $(40,41)$ and G-protein coupled receptors (42) has been reported. However, there is no evidence yet that ligand-independent EGFR signalling promotes filopodia formation. In contrast, heparin-binding EGF-like growth factor (HB-EGF), an EGFR ligand, regulates invadopodia (43) and invasion (44) via EGFR activation in some cancers. Thus, the HB-EGF/EGFR pathway is likely involved in regulating filopodia formation in SAS cells.

We found that the phosphorylation levels of c-Met were increased by EGF and decreased by AG1478 in HSC4 and SAS cells. These results suggest that EGFR activation by EGF results in transactivation of c-Met. A possible mechanism for this transactivation is that EGF/EGFR signalling upregulates HGF production, thereby releasing HGF, which stimulates c-Met phosphorylation. Ligand-activated c-Met via EGFR activation would be expected to promote cell migration. EGF/EGFR signalling affected migration in SAS cells but not HSC4 cells. Thus, the EGF/EGFR/HGF/c-Met axis may play a role in the migration of SAS cells. Another possibility is that lateral signalling from EGFR to c-Met occurs through a certain mediator, for example, c-Src (45). Such lateral signalling from the EGFR/c-Src/c-Met axis may promote lamellipodia formation and cell migration through lamellipodin upregulation in HSC4 and SAS cells, although further investigation of this is needed.

In conclusion, we showed that the HGF/c-Met and EGF/EGFR pathways increased the level of lamellipodin protein, thereby inducing cell migration via lamellipodia formation in OSCC cells. Further investigations of downstream effectors in c-Met signalling will be useful for identifying potential new therapeutic targets for OSCC patients.

\section{Acknowledgements}

The present study was edited by Textcheck English consultants. This research was supported by the Osaka University (M.N., no. 1508000001) and the Osaka Dental University (Y.O., no 217006). H.Y. is funded by the Osaka Dental University Research Funds (no.15-07).

\section{References}

1. Parkin DM, Bray F, Ferlay $\mathrm{J}$ and Pisani P: Global cancer statistics, 2002. CA Cancer J Clin 55: 74-108, 2005.

2. Xi S and Grandis JR: Gene therapy for the treatment of oral squamous cell carcinoma. J Dent Res 82: 11-16, 2003.

3. Magrath I and Litvak J: Cancer in developing countries: Opportunity and challenge. J Natl Cancer Inst 85: 862-874, 1993.
4. Kramer RH, Shen $\mathrm{X}$ and Zhou H: Tumor cell invasion and survival in head and neck cancer. Cancer Metastasis Rev 24: 35-45, 2005.

5. Ziober AF, Falls EM and Ziober BL: The extracellular matrix in oral squamous cell carcinoma: Friend or foe? Head Neck 28: 740-749, 2006

6. Berenson JR, Yang J and Mickel RA: Frequent amplification of the bcl-1 locus in head and neck squamous cell carcinomas. Oncogene 4: 1111-1116, 1989.

7. De Herdt MJ and Baatenburg de Jong RJ: HGF and c-MET as potential orchestrators of invasive growth in head and neck squamous cell carcinoma. Front Biosci 13: 2516-2526, 2008.

8. Kalyankrishna S and Grandis JR: Epidermal growth factor receptor biology in head and neck cancer. J Clin Oncol 24: 2666-2672, 2006.

9. Rørth P: Collective cell migration. Annu Rev Cell Dev Biol 25: 407-429, 2009.

10. Neiva KG, Zhang Z, Miyazawa M, Warner KA, Karl E and Nör JE: Cross talk initiated by endothelial cells enhances migration and inhibits anoikis of squamous cell carcinoma cells through STAT3/Akt/ERK signaling. Neoplasia 11: 583-593, 2009.

11. Friedl $\mathrm{P}$ and Wolf $\mathrm{K}$ : Plasticity of cell migration: A multiscale tuning model. J Cell Biol 188: 11-19, 2010.

12. Lemmon MA and Schlessinger J: Cell signaling by receptor tyrosine kinases. Cell 141: 1117-1134, 2010.

13. Jorissen RN, Walker F, Pouliot N, Garrett TP, Ward CW and Burgess AW: Epidermal growth factor receptor: Mechanisms of activation and signalling. Exp Cell Res 284: 31-53, 2003.

14. Temam S, Kawaguchi H, El-Naggar AK, Jelinek J, Tang H, Liu DD, Lang W, Issa JP, Lee JJ and Mao L: Epidermal growth factor receptor copy number alterations correlate with poor clinical outcome in patients with head and neck squamous cancer. J Clin Oncol 25: 2164-2170, 2007.

15. Ang KK, Berkey BA, Tu X, Zhang HZ, Katz R, Hammond EH, Fu KK and Milas L: Impact of epidermal growth factor receptor expression on survival and pattern of relapse in patients with advanced head and neck carcinoma. Cancer Res 62: 7350-7356, 2002.

16. Bonner JA, Harari PM, Giralt J, Cohen RB, Jones CU, Sur RK Raben D, Baselga J, Spencer SA, Zhu J, et al: Radiotherapy plus cetuximab for locoregionally advanced head and neck cancer: 5-year survival data from a phase 3 randomised trial, and relation between cetuximab-induced rash and survival. Lancet Oncol 11: 21-28, 2010

17. Fung $C$ and Grandis JR: Emerging drugs to treat squamous cell carcinomas of the head and neck. Expert Opin Emerg Drugs 15: 355-373, 2010.

18. Ohnishi Y, Yasui H, Kakudo K and Nozaki M: Cetuximabresistant oral squamous cell carcinoma cells become sensitive in anchorage-independent culture conditions through the activation of the EGFR/AKT pathway. Int J Oncol 47: 2165-2172, 2015.

19. Ohnishi Y, Yasui H, Kakudo K and Nozaki M: Regulation of cell migration via EGFR signaling in oral squamous cell carcinoma. Oncol Lett 13: 930-936, 2017.

20. Birchmeier C, Birchmeier W, Gherardi E and Vande Woude GF: Met, metastasis, motility and more. Nat Rev Mol Cell Biol 4: 915-925, 2003

21. Benvenuti S and Comoglio PM: The MET receptor tyrosine kinase in invasion and metastasis. J Cell Physiol 213: 316-325, 2007.

22. Ma PC, Maulik G, Christensen J and Salgia R: c-Met: Structure, functions and potential for therapeutic inhibition. Cancer Metastasis Rev 22: 309-325, 2003.

23. Peruzzi B and Bottaro DP: Targeting the c-Met signaling pathway in cancer. Clin Cancer Res 12: 3657-3660, 2006.

24. Hui AY, Meens JA, Schick C, Organ SL, Qiao H, Tremblay EA, Schaeffer E, Uniyal S, Chan BM and Elliott BE: Src and FAK mediate cell-matrix adhesion-dependent activation of Met during transformation of breast epithelial cells. J Cell Biochem 107: 1168-1181, 2009.

25. Henderson V, Smith B, Burton LJ, Randle D, Morris M and Odero-Marah VA: Snail promotes cell migration through PI3K/AKT-dependent Rac1 activation as well as PI3K/ AKT-independent pathways during prostate cancer progression. Cell Adhes Migr 9: 255-264, 2015.

26. Lin CW, Sun MS, Liao MY, Chung CH, Chi YH, Chiou LT, Yu J, Lou KL and Wu HC: Podocalyxin-like 1 promotes invadopodia formation and metastasis through activation of Rac1/Cdc42/ cortactin signaling in breast cancer cells. Carcinogenesis 35: 2425-2435, 2014. 
27. Rottner $\mathrm{K}$ and Stradal TE: Actin dynamics and turnover in cell motility. Curr Opin Cell Biol 23: 569-578, 2011.

28. Usatyuk PV, Fu P, Mohan V, Epshtein Y, Jacobson JR, GomezCambronero J, Wary KK, Bindokas V, Dudek SM, Salgia R, et al: Role of c-Met/phosphatidylinositol 3-kinase (PI3k)/Akt signaling in hepatocyte growth factor (HGF)-mediated lamellipodia formation, reactive oxygen species (ROS) generation, and motility of lung endothelial cells. J Biol Chem 289: 13476-13491, 2014.

29. Michael M, Vehlow A, Navarro C and Krause M: c-Abl, Lamellipodin, and Ena/VASP proteins cooperate in dorsal ruffling of fibroblasts and axonal morphogenesis. Curr Biol 20 783-791, 2010.

30. Law AL, Vehlow A, Kotini M, Dodgson L, Soong D, Theveneau E, Bodo C, Taylor E, Navarro C, Perera U, et al: Lamellipodin and the Scar/WAVE complex cooperate to promote cell migration in vivo. J Cell Biol 203: 673-689, 2013.

31. Takeshita A, Iwai S, Morita Y, Niki-Yonekawa A, Hamada M and Yura Y: Wnt5b promotes the cell motility essential for metastasis of oral squamous cell carcinoma through active Cdc42 and RhoA. Int J Oncol 44: 59-68, 2014.

32. Ma PC, Jagadeeswaran R, Jagadeesh S, Tretiakova MS, Nallasura V, Fox EA, Hansen M, Schaefer E, Naoki K, Lader A, et al: Functional expression and mutations of c-Met and its therapeutic inhibition with SU11274 and small interfering RNA in non-small cell lung cancer. Cancer Res 65: 1479-1488, 2005.

33. Wehrle-Haller B and Imhof BA: Actin, microtubules and focal adhesion dynamics during cell migration. Int J Biochem Cell Biol 35: 39-50, 2003.

34. Krause M, Leslie JD, Stewart M, Lafuente EM, Valderrama F, Jagannathan R, Strasser GA, Rubinson DA, Liu H, Way M, et al: Lamellipodin, an Ena/VASP ligand, is implicated in the regulation of lamellipodial dynamics. Dev Cell 7: 571-583, 2004.

35. Hsu PC, You B, Yang YL, Zhang WQ, Wang YC, Xu Z, Dai Y, Liu S, Yang CT, Li H, et al: YAP promotes erlotinib resistance in human non-small cell lung cancer cells. Oncotarget 7 : 51922-51933, 2016.
36. Ritter CA and Arteaga CL: The epidermal growth factor receptor-tyrosine kinase: A promising therapeutic target in solid tumors. Semin Oncol 30 (Suppl 1): 3-11, 2003.

37. Kuhlmann CR, Schaefer CA, Fehsecke A, Most AK, Tillmanns H and Erdogan A: A new signaling mechanism of hepatocyte growth factor-induced endothelial proliferation. J Thromb Haemost 3: 2089-2095, 2005.

38. Wondergem R, Ecay TW, Mahieu F, Owsianik G and Nilius B: $\mathrm{HGF} / \mathrm{SF}$ and menthol increase human glioblastoma cell calcium and migration. Biochem Biophys Res Commun 372: 210-215, 2008.

39. Tan CS, Gilligan D and Pacey S: Treatment approaches for EGFR-inhibitor-resistant patients with non-small-cell lung cancer. Lancet Oncol 16: e447-e459, 2015.

40. Yu X, Miyamoto S and Mekada E: Integrin $\alpha 2 \beta$ 1-dependent EGF receptor activation at cell-cell contact sites. J Cell Sci 113: 2139-2147, 2000.

41. Bill HM, Knudsen B, Moores SL, Muthuswamy SK, Rao VR, Brugge JS and Miranti CK: Epidermal growth factor receptordependent regulation of integrin-mediated signaling and cell cycle entry in epithelial cells. Mol Cell Biol 24: 8586-8599, 2004.

42. Wang Z: Transactivation of epidermal growth factor receptor by $\mathrm{G}$ protein-coupled receptors: Recent progress, challenges and future research. Int J Mol Sci 17: E95, 2016.

43. Díaz B, Yuen A, Iizuka S, Higashiyama S and Courtneidge SA: Notch increases the shedding of HB-EGF by ADAM12 to potentiate invadopodia formation in hypoxia. J Cell Biol 201: 279-292, 2013.

44. Ohnishi Y, Inoue H, Furukawa M, Kakudo K and Nozaki M: Heparin-binding epidermal growth factor-like growth factor is a potent regulator of invasion activity in oral squamous cell carcinoma. Oncol Rep 27: 954-958, 2012.

45. Dulak AM, Gubish CT, Stabile LP, Henry C and Siegfried JM: HGF-independent potentiation of EGFR action by c-Met. Oncogene 30: 3625-3635, 2011. 